\title{
HARVESTED ENERGY-ADAPTIVE MAC PROTOCOL FOR ENERGY HARVESTING IO'T NETWORKS
}

\author{
Hyeong-Kyu Lee, MinGyu Lee and Tae-Jin Lee \\ College of Information and Communication Engineering \\ Sungkyunkwan University, Suwon 16419, South Korea \\ lhkyulskku.edu, lmk0218@skku.edu, tjlee@skku. edu
}

\begin{abstract}
In energy harvesting IoT networks, an energy queue state of an IoT device will change dynamically and the number of IoT devices that transmit data to the IoT AP will vary in a frame. So we need a MAC protocol to adjust the frame length taking the amount of energy of IoT devices into consideration. Since the existing Framed slotted ALOHA (F-ALOHA) Medium Access Control (MAC) protocol utilizes the fixed frame size, the resource efficiency can be reduced. In this paper, we propose a Harvested Energy-adaptive Medium Access Control (HE$M A C)$ protocol where an IoT Access Point (AP) allocates slots in accordance with the number of IoT devices that try to transmit data in a frame. The proposed HE-MAC protocol improves the resource efficiency of the F-ALOHA MAC protocol. We show that the resource efficiency of the HE-MAC protocol is superior to those of the F-ALOHA MAC protocol through simulations.
\end{abstract}

\section{KEYWORDS}

Internet of Things, Energy Harvesting, Framed Slotted ALOHA, Medium Access Control.

\section{INTRODUCTION}

In Internet of Things (IoT) networks, IoT nodes have the limited battery lifetime and the batteries need to be replaced. To solve the problem, the energy harvesting technology draws much attention, since electric energy is created from the energy sources that exist in surroundings such as solar, heat, pressure, and electromagnetic wave energy [1]. The energy harvesting technology enables the sustainable use of the battery of IoT nodes in IoT networks [2]. However, in an energy harvesting IoT network, the energy states of IoT nodes change dynamically. A Computational Radio Frequency IDentification (CRFID) is an example of utilizing the energy harvesting technology. CRFID with sensing and computation functions is a new emerging technology which makes IoT devices to operate without battery [3].

According to the standard in ISO-18000-6C, the CRFID identification protocol utilizes the Framed Slotted ALOHA (F-ALOHA) Medium Access Control (MAC) protocol. CRFID tags randomly select one slot among the slots in a frame to transmit data packets [4]. The existing FALOHA MAC protocol utilizes the fixed frame length [5]. An IoT node selects one slot among the slots in a frame and transmits data to the IoT access point (AP) at the selected time slot. The number of the slots in a frame affects the throughput performance of the F-ALOHA MAC

David C. Wyld et al. (Eds) : NETCOM, NCS, WiMoNe, CSEIT, SPM - 2015

pp. 51-58, 2015. () CS \& IT-CSCP 2015

DOI : $10.5121 /$ csit.2015.51605 
protocol. Since the amount of energy stored in a battery may change in a frame, some IoT nodes with insufficient energy to transmit data may exist. If the number of slots is more than the number of the IoT nodes in a frame, the resource efficiency is reduced due to the unused slots. If the number of slots is less than the number of the IoT nodes in a frame, the resource efficiency is also reduced due to the collided slots. Thus, a new MAC protocol to adjust the number of slots according to the energy state in a frame is required.
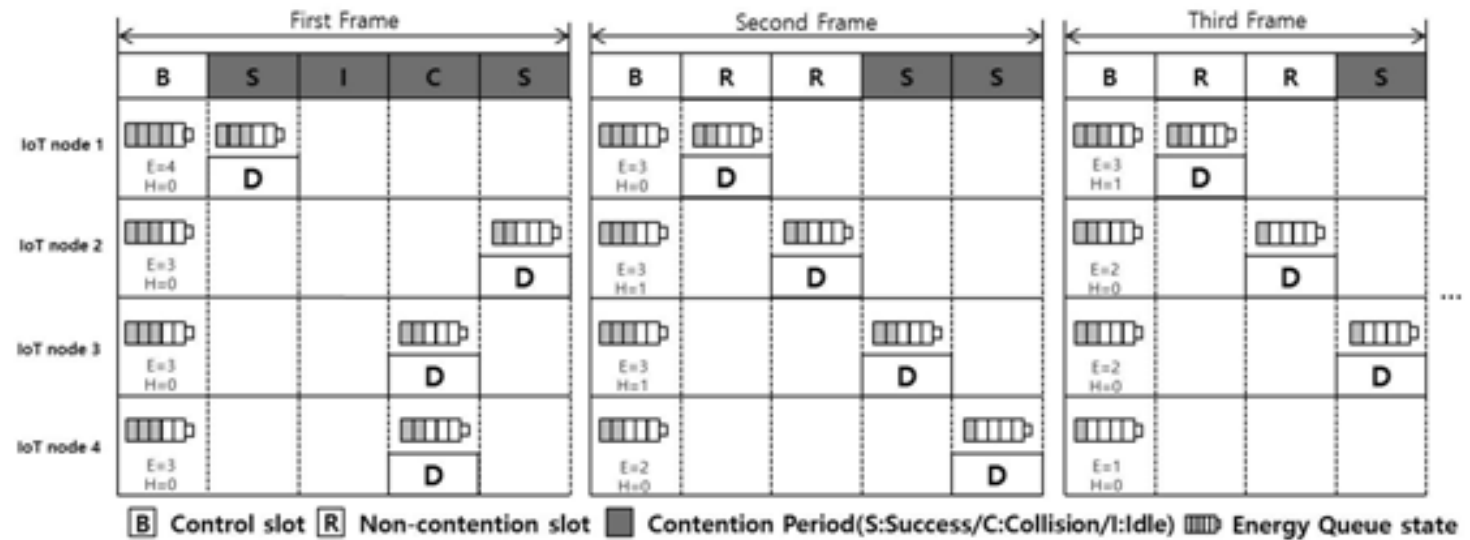

Figure 1. An example of the proposed the HE-MAC protocol with $N(=4)$ and $E_{m i n}$.

In this paper, we propose a harvested energy-adaptive MAC (HE-MAC) protocol to adjust the frame length taking the energy states of IoT nodes into account. The proposed HE-MAC protocol alleviates the reduction of the resource efficiency of the F-ALOHA MAC protocol. The detailed explanation of the proposed HE-MAC protocol is presented in Section 2. In Section 3, we compare the throughput performance of the proposed HE-MAC with that of the existing FALOHA MAC protocol. Finally, we conclude in Section 4.

\section{RELATED WORK}

In [10], the S-MAC protocol is proposed to reduce energy consumption. The protocol utilizes periodic listen and sleep scheme, so each node does not need to listen at all the times. Also, the nodes exchange the Request To Send (RTS) and Clear To Send (CTS) before actual data transmission to avoid collision and overhearing. However, the protocol does not consider energy harvesting technology. The authors of [11] propose a duty-cycle optimization scheme by finding the minimum length of the non-active period satisfying Energy Neutral Operation (ENO) of energy harvesting nodes. Since the scheme utilizes F-ALOHA MAC protocol, it does not adapt the frame length according to the number of the nodes transmitting data packets during the active period.

The design of a MAC protocol such as TDMA, and F-ALOHA in an energy harvesting circumstance is presented in [12]. Also, the trade-off relationship between the delivery efficiency and the time efficiency is investigated. But the work does not consider variability in the number of nodes transmitting data packets due to fluctuation of the energy level of the individual nodes. In [13], the authors present a fair polling scheme for energy harvesting wireless sensor networks. The scheme improves the fairness by considering priority using the harvesting rate of the nodes. The scheme focuses on only fairness, but improvement of the throughput is not considered. 


\section{PROPOSED HE-MAC PROTOCOL}

In this section, we present the proposed HE-MAC protocol. We consider an energy harvesting network which consists of an IoT AP and $N$ IoT nodes. The IoT nodes transmit data with the energy queue states of IoT nodes. We assume that the energy stored in an energy queue is composed of energy blocks. The maximum number of energy blocks that can be stored in a battery is $E_{\max }$. The energy queue state can be reduced with the unit of an energy block when an IoT node transmits a data packet. An IoT node harvests an energy block with the energy harvesting probability $P_{h}$ in a slot. Let $E_{\min }$ denote the energy threshold. The energy queue state of IoT nodes has to exceed $E_{\min }$ in a frame to transmit data. For example, if $E_{m i n}=1$, the IoT node that the energy queue state is one does not transmit data.

A frame consists of the control slot, the non-contention slot, and the contention slot. An IoT AP broadcasts a control packet to the IoT nodes at the control slot. The control packet includes the number of non-contention slots, the number of contention slots, and the allocation information for the non-contention slots in the $i$ th frame. A non-contention slot is allocated to only one IoT node that transmits data successfully in the (i-1)th frame and exceeds $E_{\min }$. The contention slot is for the IoT nodes that collide with one another in the $(i-1)$ th frame or for those not allocated noncontention slots. At the end of a frame, the contention slots can be classified as success slots, collision slots, and idle slots. The IoT AP receiving data packets with energy state information of the IoT nodes decides to allocate non-contention slots in the $i$ th frame according to the information of the energy state of the IoT nodes. After deciding which IoT nodes transmit at noncontention slots, the IoT AP estimates the number of the IoT nodes trying to transmit at contention slots according to the information of the energy state of IoT nodes and the collision slots in the $(i-1)$ th frame.

IoT nodes may receive control packets at the control slot. If IoT nodes succeed in transmission and the energy level exceeds $E_{\min }$ in the $(i-1)$ th frame, they are allocated non-contention slots in the $i$ th frame, they transmit their data packets at their allocated non-contention slots. If collision does not happen in the non-contention slot, the IoT nodes transmitting in the non-contention slots will be continuously allocated in the non-contention slots as long as the energy states of the IoT nodes exceed $E_{\min }$. If the energy level of an IoT node does not exceed $E_{\min }$, the IoT node harvests energy without transmitting of data packets. If IoT nodes collide in the $(i-1)$ th frame and the energy levels exceed $E_{\min }$ at the start of the $i$ th frame, they each randomly select one of the contention slots and try to transmit at the selected the contention slots in the $i$ th frame.

Fig. 1 shows an example of the proposed HE-MAC protocol with $N$ nodes and $E_{\min }(=1)$. Let $E$ and $H$ denote the energy queue state of an IoT node and the amount of harvested energy in the previous frame. In the first frame, all slots except the control slot are contention slots. The IoT terminals randomly select one of the four contention slots and transmit data packets to the IoT AP at the selected contention slot. The IoT node 1 and the IoT node 2 successfully transmit data packets in the first frame and consume one energy block. The IoT node 3 and the IoT node 4 collide with each other at the third slot and use energy blocks. After transmission, the amount of the remaining energy blocks of the IoT node 1 and the IoT node 2 is 3 and 2 . The energy state of the IoT node 3 and the IoT 4 is 2 after collision at the third slot. Since the energy levels of the IoT nodes exceed $E_{\min }(=1)$, they can transmit data in the second frame.

In the second frame, the IoT nodes update energy states by adding the harvested energy during the first frame. The energy states of the IoT node 1 , node 2 , node 3 , and node 4 are $3,3,3$, and 2 , respectively at the beginning of the second frame. The IoT node 1 and the IoT node 2 transmit 
data packets successfully in the allocated non-contention slots in the second frame since they succeed in transmission and the energy levels exceed $E_{\min }$ in the first frame. The IoT node 3 and the IoT node 4 succeed in transmission of the data packets by selecting different slots with each other. Since the IoT node 4 does not have enough energy after data transmission in the second frame, the energy queue state information indicates that it does not transmit its data packet in the next frame.

In the third frame, the IoT nodes update the energy states as in the second frame. If any IoT node does not transmit its data packet at the contention slot in the next frame, one of the IoT nodes that succeed in transmission in the previous frame transmits its data packet at the contention slot. The IoT node 1 and the IoT node 2 succeed in data transmission in the non-contention slots and the IoT node 3 transmits data successfully at the contention slot. However, the IoT node 4 does not transmit data, and harvests energy during the third frame. The IoT node 2 and the IoT node 3 do not transmit data in the next frame since the energy states do not exceed $E_{\min }$. If the energy state of the IoT node 4 exceeds $E_{\min }$, the IoT node 4 will transmit its data packet at a contention slot in the next frame.

Table 1. Parameters of Simulation.

\begin{tabular}{|c|c|}
\hline Parameters & Value \\
\hline Energy harvesting probability $\left(P_{h}\right)$ & $0.01-1$ \\
\hline Number of IoT nodes $(N)$ & $10-150$ \\
\hline Energy threshold $\left(E_{\min }\right)$ & 1 \\
\hline Size of the energy queue $\left(E_{\max }\right)$ & 5 \\
\hline Initial state of the energy queue $\left(E_{\text {init }}\right)$ & 5 \\
\hline
\end{tabular}

The proposed HE-MAC protocol adjusts the frame size according to the number of IoT nodes trying to transmit, while the F-ALOHA MAC protocol utilizes the fixed frame size. If the number of IoT nodes decreases, there may be unused slots in the F-ALOHA MAC protocol. However, the proposed HE-MAC protocol allocates slots according to the amount of stored energy states of the IoT nodes, and the number of wasted slots can be reduced.

\section{SIMULATION}

In this section, we present the resource efficiency of the proposed HE-MAC protocol. The FALOHA MAC protocol utilizes the fixed frame size in which the number of contention slots is the same as $N$. The parameters are summarized in Table $1 . E_{\text {init }}$ denotes the initial state of the energy queue. We assume that the IoT AP knows all the energy queue states of the IoT nodes, and the number of IoT nodes does not change.

Fig. 2 presents throughput for varying harvesting probability with different $N$. The throughput increases as the harvesting probability increases. Since the harvested energy increases as the harvesting probability increases, the number of IoT nodes which have enough energy to transmit data packets increases. So, the IoT AP can allocate non-contention slots to the IoT nodes consistently. Since the success slots increases as the number of non-contention slots increases, the throughput also increases. However, the number of non-contention slots does not exceed $N$, thus the throughput is eventually saturated. When $N=150$, the throughput performances are similar for almost any harvesting probabilities since the maximum frame size when $N=150$ is larger than those of others, and the harvested energy of IoT nodes when $N=150$ is larger than those of others. 
Therefore, the throughput performance when $N=150$ can be easily saturated even if the harvesting probability is low.

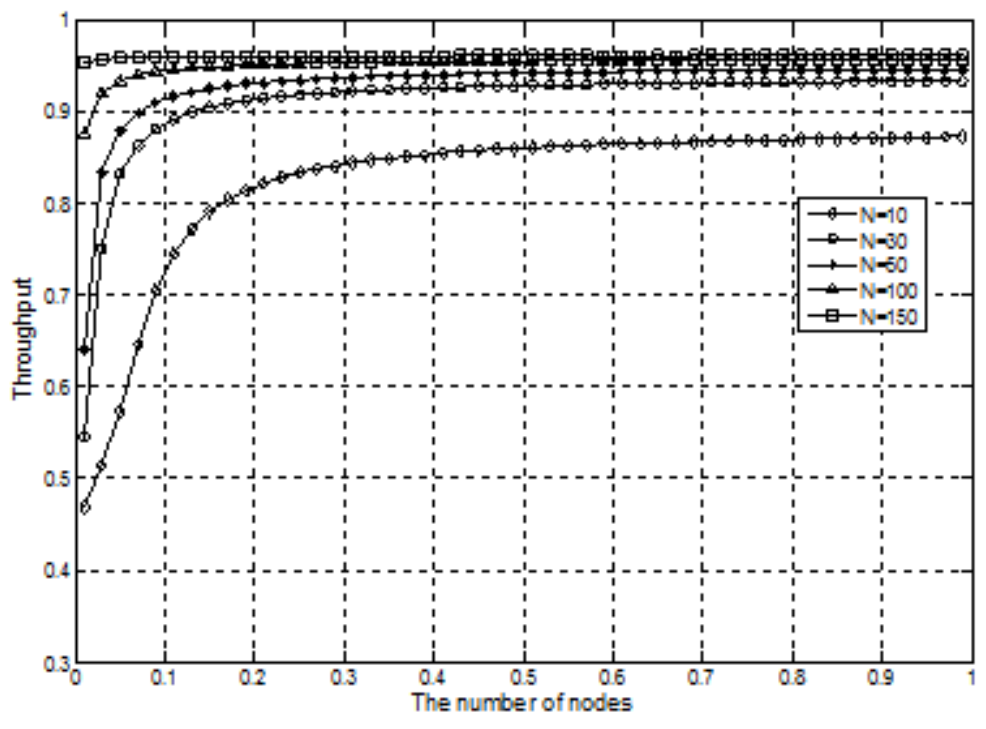

Figure 2. Throughput for varying harvesting probabilities with different $N$.

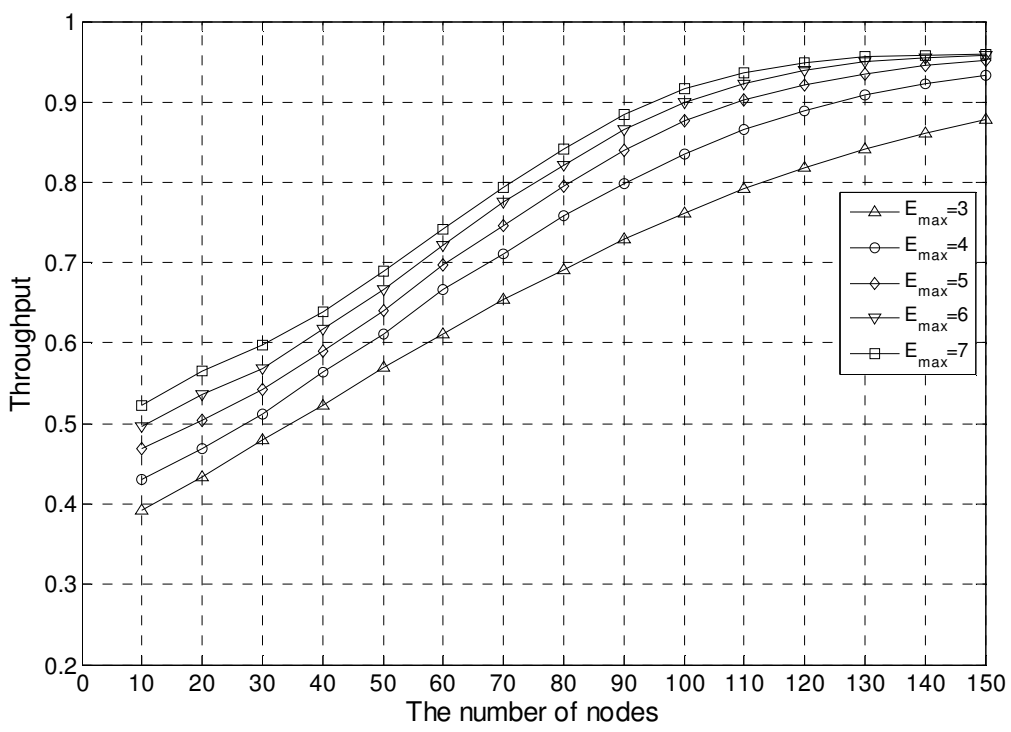

Figure 3. Throughput for varying number of IoT nodes with different $E_{\max }$ and $P_{h}(=0.01)$.

Fig. 3 shows the throughput for varying number of nodes with different $E_{\max }$ and $P_{h}$. The battery of an IoT node can store more energy as the size of the energy queue increases. If an IoT node can store more energy, it can transmit data packets consistently at the non-contention slot until the energy state of the IoT node becomes lower than $E_{\min }$. Since the number of IoT nodes that successfully transmits data packets consistently at the non-contention slot increases, the throughput increases according to the size of energy queue. In the case that the energy harvesting 
probability is high, the energy queue is charged quickly then the size of the energy queue does not much affect the throughput performance.

In Fig. 4, we compare the throughput performances of the HE-MAC protocol to those of the FALOHA MAC protocol by changing $N$. The throughputs of the two protocols increase as $N$ increases. If the energy harvesting probability increases, the number of IoT nodes that harvested energy increases in a frame. Since the number of IoT nodes transmitting data packets at the noncontention slot increases, the throughputs increase. When $N=10$, as the energy harvesting probability increases from 0.01 to 0.09 , the throughput increases from 0.47 to 0.84 . Since the FALOHA MAC protocol utilizes fixed frame size, it does not adjust the number of slots according to the number of IoT nodes transmitting data packets. So, the throughputs of F-ALOHA MAC protocol are less than those of the HE-MAC protocol.

\section{CONCLUSION}

In this paper, we proposed the HE-MAC protocol that adjusts the frame length in accordance with the number of IoT nodes trying to transmit in a frame. Since the existing F-ALOHA MAC protocol utilizes the fixed frame size, it does not adjust the number of IoT nodes that try to transmit data packets according to the energy states of IoT nodes. The HE-MAC protocol can allocate the radio resources taking into account the energy states of IoT nodes. Thus, our proposed MAC protocol alleviates the reduction of the resource efficiency of the F-ALOHA MAC protocol. The simulation results show that our proposed HE-MAC protocol increases throughput performance than the F-ALOHA MAC protocol as the probability of energy harvesting and the number of IoT nodes increase.

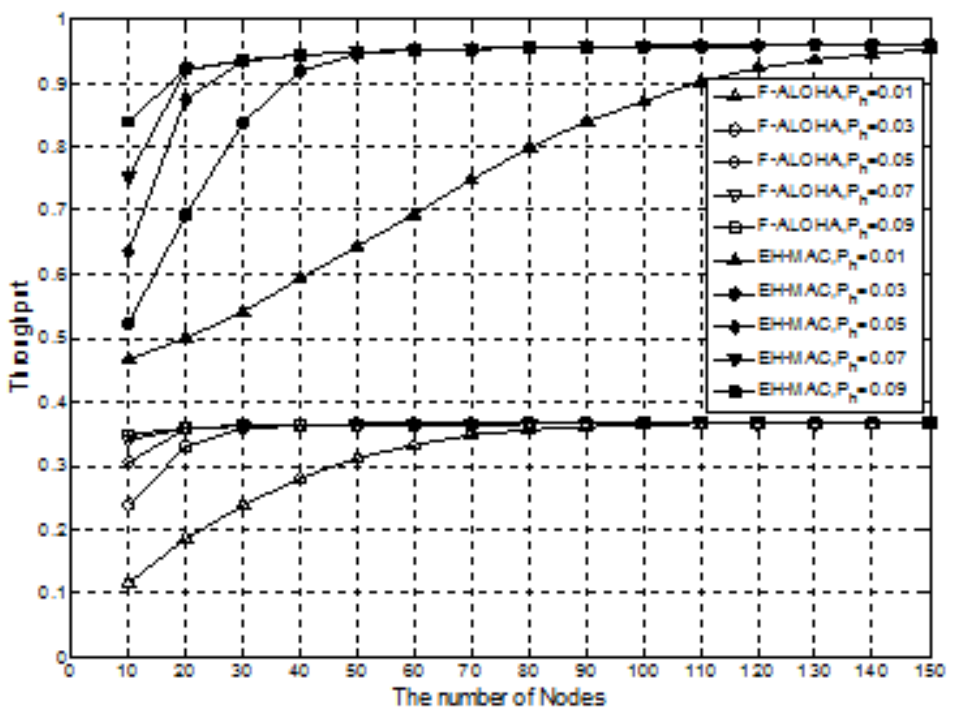

Figure 4. Throughput for varying number of IoT nodes.

\section{ACKNOWLEDGEMENT}

This work was supported by the National Research Foundation of Korea (NRF) grant funded by the Korean government (MSIP)(2014R1A5A1011478, 2015R1A2A2A01004067). 


\section{REFERENCES}

[1] S. Sudevalayam and P. Kulkarni, "Energy Harvesting Sensor Nodes: Survey and Implications Energy Harvesting Sensor Nodes: Survey and Implications," IEEE Communications Surveys \& Tutorials, vol. 13, no. 3, pp. 443-461, Third Quarter, 2011.

[2] H. Li, J. Xu, R. Zhang, and S. Cui, "A General Utility Optimization Framework for EnergyHarvesting based Wireless Communications," IEEE Communications Magazine, vol. 53, no. 4, pp. 79-85, Apr. 2015.

[3] W. Yang, D. Wu, M. J. Hussain, and L. Lu "Wireless Firmware Execution Control in Computational RFID Systems," in Proc. of IEEE RFID, pp. 129-136, Apr. 2015.

[4] A. Wickramasinghe and D. C. Ranasinghe, "Ambulatory Monitoring Using Passive Computational RFID Sensors,” IEEE Sensors Journal, vol. 15, no. 10, pp. 5859-5869, Oct. 2015.

[5] J. E. Wieselthier, A. Ephremides, and L. A. Michaels, "An Exact Analysis and Performance Evaluation of Framed ALOHA with Capture," IEEE Transactions on Communications, vol. 37, no. 2, pp. 125-137, Feb. 1989.

[6] J. Vales-Alonso, F. J. Parrado-Garcia, and J. J. Alcaraz, "Analytical Computation of the Mean Number of Tag Identifications during a Time Interval in FSA," IEEE Communications Letters, vol. 18, no. 11, pp. 1923-1926, Nov. 2014.

[7] O. Omni, A. Wong, A. J. Burdett, and C. Toumazou, "Energy Efficient Medium Access Protocol for Wireless Medical Body Area Sensor Networks," IEEE Transactions on Biomedical Circuits and Systems, vol. 2, no. 4, pp. 251-259, Dec. 2008.

[8] M. Gorlatova, P. Kinget, I. Kymissis, D. Rubenstein, X. Wang, and G. Zussaman, "Challenge: Ultralow-power Energy-harvesting Active Neworked Tags(EnHANTs)," in Proc. of MobiCom, pp. 253260, Sep. 2009.

[9] J. Liu, H. Dai, and W. Chen, "On Throughput Maximization of Time Division Multiple Access with Energy Harvesting Users," IEEE Transactions on Vehicular Technology, to be published.

[10] W. Ye, J. Heidemann, D. Estrin, "An Energy-Efficient MAC Protocol for Wireless Sensor Networks ,”in Proc. of INFOCOM, pp. 1567-1576, Jun. 2002.

[11] O. Briante, A. M. Mandalari, A. Molinaro, G. Ruggeri, J. Alonso-Zarate, and F. Vazquez-Gallego, "Duty-Cycle Optimization for Machine-to-Machine Area Networks based on Frame Slotted-ALOHA with Energy Harvesting Capabilities," in Proc. of EW, pp. 1-6, May. 2014.

[12] F. Iannello, O. Simeone, and U. Spagnolini, "Medium Access Control Protocols for Wireless Sensor Networks with Energy Harvesting," IEEE Transactions on Communications, vol. 60, no. 5, pp. 13811389, May. 2012.

[13] M. Kunikawa, H. Yomo, K. Abe, T. Ito, "A Fair Polling Scheme for Energy Harvesting Wireless Sensor Networks,"in Proc. of VTC spring, pp. 1-5, May. 2015.

[14] Y. He, X. Cheng, W. Peng, and G. L. Stuber, "A Survey of Energy Harvesting Communications: Models and Offline Optimal Policies," IEEE Communications Magazine, vol. 53, no. 6, pp. 79-85, Jun. 2015. 


\section{AUTHORS}

Hyeong-Kyu Lee received B.S. degree in electronics communication engineering from Hanyang University, Korea in 2013. He is currently working toward his M.S. degree in the College of Information and Communication Engineering at Sungkyunkwan University, Suwon, Korea since March 2015. His research interests include energy harvesting, M2M communications, IoT and Medium Access Control (MAC).

Mingyu Lee received the B.S. degree in electronics engineering from Kwangwoon University, Korea in 2009 and the M.S. degree in mobile systems engineering from Sungkyunkwan University, Suwon, Korea in 2012. He is currently pursuing his Ph.D. degree in IT convergence at Sungkyunkwan University since March 2012. His research interests include cognitive radio networks, wireless LANs, ad hoc networks, vehicular networks, energy harvesting, M2M communications and IoT.

Tae-Jin Lee received his B.S. and M.S. in electronics engineering from Yonsei University, Korea in 1989 and 1991, respectively, and the M.S.E. degree in electrical engineering and computer science from University of Michigan, Ann Arbor, in 1995. $\mathrm{He}$ received the Ph.D. degree in electrical and computer engineering from the University of Texas, Austin, in May 1999. In 1999, he joined Corporate R \& D Center, Samsung Electronics where he was a senior engineer. Since 2001, he has been Professor in the College of Information and Communication Engineering at
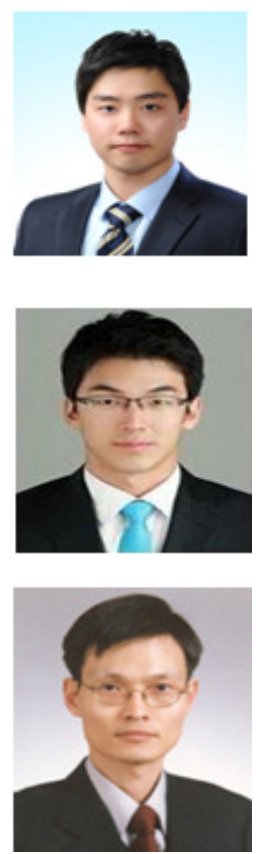
Sungkyunkwan University, Korea. He was a visiting professor in Pennsylvania State University from 2007 to 2008. His research interests include performance evaluation, resource allocation, Medium Access Control (MAC), and design of communication networks and systems, wireless LANs/PANs, vehicular networks, energy-harvesting networks, IoT, ad hoc/sensor/RFID networks, and next generation wireless communication systems. He has been a voting member of IEEE 802.11 WLAN Working Group, and is a member of IEEE and IEICE. 